\title{
Marc RENAUD
}

Sociologue, département de sociologie, Université de Montréal

(1977)

\section{"Réforme ou illusion? \\ Une analyse des interventions de l'État québécois dans le domaine de la santé”}

Un document produit en version numérique par Jean-Marie Tremblay, bénévole, Professeur sociologie au Cégep de Chicoutimi Courriel: jean-marie_tremblay@uqac.ca

Dans le cadre de "Les classiques des sciences sociales"

Site web: http://classiques.uqac.ca/

Une bibliothèque fondée et dirigée par Jean-Marie Tremblay, sociologue

Une collection développée en collaboration avec la Bibliothèque Paul-Émile-Boulet de l'Université du Québec à Chicoutimi Site web: http://bibliotheque.uqac.ca/ 
Cette édition électronique a été réalisée Jean-Marie Tremblay, bénévole, professeur de soins infirmiers retraitée de l'enseignement au Cégep de Chicoutimi

Courriel: jean-marie_tremblay@uqac.ca

à partir du texte de :

Marc Renaud, "Réforme ou illusion? Une analyse des interventions de l'État québécois dans le domaine de la santé”.

Un article publié dans la revue Sociologie et sociétés, vol. 9, no 1, avril 1977, pp. 127-152. Montréal : Les Presses de l'Université de Montréal.

M. Marc Renaud est sociologue au département de sociologie de l’Université de Montréal.

[Autorisation formelle de l'auteur accordée le 7 mai 2006.]

Courriel : marc.renaud@gmail.com

Polices de caractères utilisée :

Pour le texte: Times New Roman, 14 points.

Pour les citations : Times New Roman 12 points.

Pour les notes de bas de page : Times New Roman, 12 points.

Édition électronique réalisée avec le traitement de textes Microsoft Word 2004 pour Macintosh.

Mise en page sur papier format : LETTRE (US letter), 8.5'’ x 11'’)

Édition numérique réalisée le 24 février 2007 à Chicoutimi, Ville de Saguenay, province de Québec, Canada. 


\section{Marc Renaud}

Sociologue, département de sociologie, Université de Montréal

\section{"Réforme ou illusion ? Une analyse des interventions de l'État québécois dans le domaine de la santé”.}

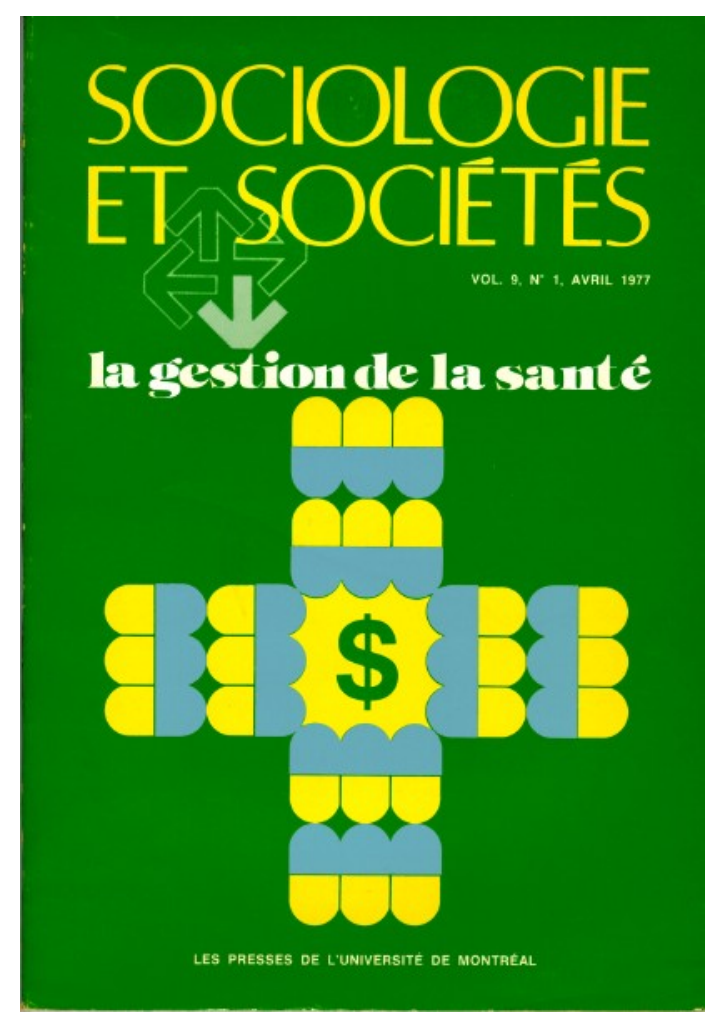

Un article publié dans la revue Sociologie et sociétés, vol. 9, no 1, avril 1977, pp. 127-152. Montréal : Les Presses de l'Université de Montréal. 


\section{Table des matières}

$\underline{\text { Introduction }}$

Évolution historique

Le rapport de la commission d'enquête sur la santé et le bien-être social (commission Castonguay-Nepveu)

La réforme

Les résultats de la réforme

De la stratégie des réformes

De l'écart entre les idéaux et la réalité de la réforme

$\underline{\text { Conclusion }}$

Résumé 
Marc Renaud,

"Réforme ou illusion? Une analyse des interventions de l'État québécois dans le domaine de la santé”. *

Un article publié dans la revue Sociologie et sociétés, vol. 9, no 1, avril 1977, pp. 127-152. Montréal : Les Presses de l'Université de Montréal.

\section{Introduction}

\section{Retour à la table des matières}

Un curieux phénomène s'est produit au Québec au cours de la dernière décennie. Des interventions de l'État visant à résoudre une multitude de " problèmes " et de " crises » sociales ont donné lieu à des espoirs et des réorganisations sans précédent au Canada. Ces réformes, toutefois, ont très souvent exacerbé les "problèmes » et les « crises » qu'elles devaient résoudre.

Dans toutes les provinces canadiennes, depuis le milieu des années 1950, les dépenses publiques, le nombre et les types d'interventions des gouvernements provinciaux ont progressé de façon à peu près comparable. Mais, au Québec, cette expansion de l'État fut accompagnée d'une telle rhétorique social-démocrate, de la promulgation d'ob-

Cet article est une version résumée et désespérément simplifiée d'une thèse de doctorat (The Political Economy of the Quebec State Interventions in Health : Reform or Revolution ?, University of Wisconsin, Madison, 1976) à être publiée sous peu. Trop de personnes m'ont fourni des renseignements ou m'ont fait des commentaires à propos de ma thèse pour que je puisse tous les remercier ici. Je voudrais toutefois remercier mes collègues Paul Bernard, Luciano Bozzini et Jacques Dofny pour leurs critiques sur la première version de cet article. Comme je n'ai pas toujours pris en considération leurs critiques sévères, ils peuvent avoir bonne conscience d'être en désaccord. 
jectifs réformistes d'une telle ampleur et d'un tel autoritarisme législatif, que les interventions du gouvernement québécois sont perçues par plusieurs comme étant uniques au Canada. Et pourtant, dans la plupart des cas, les résultats tangibles des réformes québécoises ne différent pas de ceux qui furent obtenus ailleurs. Malgré la proclamation d'objectifs beaucoup plus fondamentaux et en dépit de l'utilisation beaucoup plus systématique des pouvoirs étatiques de réorganisation et de coercition, les réformes initiées par le gouvernement québécois sont souvent ni plus (et ni moins) importantes et profondes que celles introduites dans les autres provinces canadiennes. En réalité, c'est davantage un style de gestion ou de prévention des "crises » ou, en d'autres mots, une stratégie de réforme, qui est particulier au Québec, non les changements eux-mêmes.

Les principales réformes, au cours de ce qui fut appelé la « Révolution tranquille ", ont presque toutes suivi le même cheminement et reflété la même stratégie de réforme. Dans un premier temps, pour faire face à des malaises sociaux évidents ou à de fortes pressions populaires, le gouvernement québécois a presque toujours suscité les attentes et les espoirs les plus grands. Ainsi, contrairement a ce qui se produit dans les autres provinces, des commissions d'enquête ou d'autres agents de formulation de politiques ont élaboré - et souvent avec suffisamment de détails pour être convaincants - des politiques axées sur les grands idéaux socio-démocratiques de notre époque : égalité des opportunités, défense du patrimoine, propriété collective des ressources naturelles, démocratisation de l'éducation, participation décisionnelle et consultative des citoyens et des travailleurs, décentralisation et régionalisation des pouvoirs de décision, médecine globale, etc.

Dans un second temps, une fois les politiques formulées et les attentes créées, le gouvernement introduit, à une vitesse foudroyante, des réorganisations globales très ambitieuses en utilisant presque uniquement son pouvoir de coercition législatif, sans débat public prolongé, expérience pilote ou autres procédures de changement graduel. Ici encore, on constate un style de gestion politique radicalement différent des provinces anglophones ou expériences pilotes et autres procédures ad hoc (« muddling through procedures ») sont privilégiées. 
Dans un troisième temps, quelques années plus tard, on découvre que ces réformes sont des échecs, non seulement parce qu'elles apportent peu de solutions concrètes aux problèmes sociaux qu'elles promettaient de résoudre, mais aussi parce qu'elles sont très éloignées des idéaux socio-démocratiques qu'elles devaient atteindre. L'écart souvent gigantesque entre les idéaux et les objectifs mis de l'avant et leur opérationnalisation concrète constitue ainsi le ferment d'une nouvelle série de « crises » de plus en plus compliquées dans le futur.

Ce phénomène se produisit pour les réformes de l'éducation et de l'économie dans les années 1960 et le même processus se déroule, depuis 1970, dans ce qu'il est convenu d'appeler le « secteur de la santé ».

Dans le domaine de la santé, ce furent les rapports de la Commission Castonguay-Nepveu qui d'abord suscitèrent attentes et espoirs. En exigeant la création d'une nouvelle médecine, la cogestion des organisations de soins par les usagers et les travailleurs, la décentralisation du processus de décision et une certaine égalisation du statut des « professionnels » de la santé, on promettait de rationaliser et de démocratiser en profondeur le secteur de la santé et, en définitive, d'améliorer l'état de santé de la population. Suite aux recommandations de cette Commission, après que le plan d'assurance-maladie obligatoire et universelle eut été adopté en 1970, le gouvernement légiféra, en moins de quatre ans, des réformes dans le domaine des professions, de l'organisation des soins et de l'administration des services de santé sur une échelle, sur papier, sans parallèle en Amérique du Nord. Mais, entre les recommandations d'une Commission d'enquête, les textes de lois, et leur application concrète, peuvent se glisser des déviations et des récupérations qui sont la négation même des idéaux initialement prônés. C'est, comme nous le verrons, largement ce qui s'est produit. Les apparences du changement constituent le meilleur masque pour la perpétuation du statu quo. En fait, malgré tous les discours, les réformes québécoises ne dépassèrent guère en importance les changements introduits dans les autres provinces, souvent par des méthodes moins autoritaires et avec des objectifs moins ambitieux qu'au Québec. 
Dans cet article, nous ferons d'abord un survol historique de l'évolution du secteur de la santé au Québec jusqu'en 1970. Puis, nous verrons qu'au-delà de sa volonté de rationalisation bureaucratique, la Commission Castonguay-Nepveu promettait une réforme en profondeur, une véritable réforme "révolutionnaire » - pour employer l'expression d'André Gorz ${ }^{1}$ - du secteur de la santé au Québec. Ensuite, nous décrirons brièvement la réforme telle qu'elle apparaît dans les textes de lois. Enfin, nous tenterons un premier bilan, qui indique un décalage substantiel entre les idéaux proclamés et la réalité, entre ce qui fut promis et ce qui fut fait, et une grande similitude entre les réformes québécoises et celles introduites ailleurs.

Si cette description est juste, deux questions analytiques en découlent. Premièrement, comment se fait-il qu'au Québec, contrairement aux autres provinces, des idéaux socio-démocratiques soient constamment mis de l'avant et que l'État essaie de les atteindre en utilisant au maximum son pouvoir de coercition législatif ?. Après avoir examiné les différentes réponses possibles, nous concluerons que cette situation renvoie aux caractéristiques propres aux rapports de classes au Québec. La deuxième question est de savoir pourquoi ces idéaux ne furent pas atteints même si, en apparence, l'État les soutenait. Nous soutiendrons ici l'hypothèse que des contraintes structurelles empêchent l'État dans une société capitaliste d'agir dans une direction « révolutionnaire ».

\section{ÉVOLUTION HISTORIQUE}

$\underline{\text { Retour à la table des matières }}$

Jusqu'en 1960, le secteur de la santé au Québec évolua presque uniquement en fonction des forces du marché, en réponse aux changements dans la technologie médicale, à la demande croissante par la population de soins curatifs et aux intérêts des principaux entrepreneurs dans le domaine, les médecins, les communautés religieuses et les fabricants de médicaments et d'équipements hospitaliers. Le sec-

1 André Gorz, Stratégie ouvrière et néo-capitalisme, Paris, Seuil, 1964. 
teur de la santé n'était alors presque pas réglementé par l'État. Celui-ci n'intervint qu'au minimum, soit pour soutenir les forces du marché - et notamment les hôpitaux qui, au moment de la Grande Dépression, étaient presque en faillite (Loi de l'Assistance Publique) -, soit parce que des facteurs extérieurs au marché imposaient à l'État d'agir (v.g. santé publique, santé mentale). L'ordre social traditionnel d'un marché privé, camouflé sous le concept de charité, fut ainsi maintenu jusque vers 1960 malgré les boule versements technologiques et organisationnels considérables que subit l'industrie de la santé après la guerre. Les médecins, dont le statut social ne le cédait qu'à celui des prêtres, dominaient totalement la hiérarchie professionnelle et ils commandaient l'allocation des tâches dans la division du travail des soins aux malades. De plus, les médecins spécialisés en médecine préventive (ou, plus précisément, en santé publique) contrôlaient le Ministère québécois de la santé dont l'organisation des unités sanitaires était la principale responsabilité ${ }^{2}$. Le Collège des Médecins et Chirurgiens de la Province de Québec surveillait la pratique médicale, dont l'archétype était l'entrepreneur indépendant, payé à l'acte, et sans contrainte autre que professionnelle. Les communautés religieuses possédaient et géraient les principaux hôpitaux et la formation du personnel paramédical ${ }^{3}$. Plusieurs compagnies d'assurance se partageaient le marché

2 Fondées en 1933, les unités sanitaires dispensaient les services traditionnels de santé publique (vaccination, soins pré-nataux, etc.). En 1936, un Ministère de la Santé fut créé principalement pour administrer ces unités sanitaires ; ce fut sa principale responsabilité jusqu'à l'introduction du programme d'assurance-hospitalisation en 1960. Même en 1966, 71\% du personnel du Ministère était engagé dans des activités de santé publique. Alors que $59.3 \%$ des cadres du Ministère étaient, en 1968, des médecins, seulement $7.4 \%$ des cadres du Ministère des Affaires Sociales en 1974 étaient médecins.

3 On a estimé qu'en 1960, les communautés religieuses étaient propriétaires de 42\% des hôpitaux de la province, avec les deux tiers des lits hospitaliers. En janvier 1972, elles ne possédaient que 37 hôpitaux (i.e. 13\% des hôpitaux), souvent les plus isolés et marginaux. Dans tous les cas, les hôpitaux sont maintenant gérés presqu'en totalité par des laïcs. De plus, alors que les communautés religieuses contrôlaient 31 des 50 écoles de nursing en 1960, elles perdirent ce contrôle avec la réforme de l'éducation. (Jean-Pierre Alix, "Social change in the health institutions from 1960 to 1972 », communication présentée au séminaire intitulé « Social change in contemporary Quebec » du département de sociologie de l'Université de Toronto, janvier 1973). 
restreint de l'assurance-maladie privée ${ }^{4}$. Bref, malgré la complexité croissante de la division du travail ${ }^{5}$, malgré le développement phénoménal de l'appareil hospitalier ${ }^{6}$, malgré le déclin progressif de l'action sanitaire sur les maladies infectieuses ${ }^{7}$, et malgré la montée du coût des soins curatifs ${ }^{8}$, l'organisation traditionnelle des services de santé fut maintenue jusqu'en 1960 et presque intégralement jusqu'en 1970.

C'est le gouvernement fédéral qui, par ses programmes à frais partagés, fut, au départ, le principal agent de changement. Après que plusieurs commissions d'enquête eurent recommandé de faire porter par l'État le financement des soins de santé et après des conférences fédérales-provinciales tumultueuses, la Chambre des Communes à Ottawa vota l'assurance-hospitalisation en 1957 et l'assurance-maladie en

4 Près de 250 compagnies (dont les plus importantes étaient la Croix-Bleue et Les Services de Santé du Québec) assuraient une très faible proportion de la population. En 1964, par exemple, 43.1\% de la population avait une forme quelconque d'assurance-maladie (par comparaison à $70.9 \%$ en Ontario) et, parmi ceux-ci, seulement $40.7 \%$ avaient une couverture relativement complète (78.8\% en Ontario).

5 Par exemple, alors qu'il y avait un médecin pour chaque autre « professionnel de la santé » en 1921, ce rapport était de un à six en 1971. Le nombre de médecins spécialistes a atteint 62\% en 1970. 1.7\% de la population active travaillait dans le secteur de la santé en 1921 contre $2.8 \%$ en 1941 et $6.2 \%$ en 1971.

6 Voici certaines indications de cette croissance. Excluant les hôpitaux psychiatriques et les sanatoriums, la capacité théorique du Québec en lits hospitaliers par mille habitants passa de 3.26 en 1932 à 5.96 en 1970. 37.4 personnes par mille habitants sont admises dans ces hôpitaux en 1932, contre 123.3 en 1970. 41.2\% des naissances ont lieu dans les hôpitaux en 1948 contre 95\% en 1962.

7 Le déclin est très net. Par exemple, la mortalité attribuable à la tuberculose (environ 200 par mille en 1900) disparaît presque. C'est la même chose pour la fièvre typhoïde, la dysenterie, la diphtérie, la coqueluche, les oreillons et la pneumonie.

8 Par exemple, 5.3\% du Produit National Brut Canadien était affecté au secteur de la santé en 1960, contre 7.1\% en 1970. Suite à l'introduction des programmes d'assurance-hospitalisation, d'assistance médicale et d'assurance-maladie, c'est l'État qui assumera graduellement une fraction croissante de ces dépenses. Ainsi, alors que le gouvernement québécois dépensait \$1.11 par habitant pour la santé publique en 1949-50, il dépensera \$5.35 en 1971-72 ; pour les soins médicaux, ces dépenses passent de \$0.12 par tête en 1949-50 à 57.00 en 1971-72 ; pour les soins hospitaliers, \$11.08 à \$146.42. 
1966. Par ces programmes, le gouvernement fédéral assume, à certaines conditions, environ $50 \%$ des coûts dans les provinces participantes selon une formule qui favorise les provinces les plus pauvres économiquement.

Plusieurs facteurs expliquent la volonté du gouvernement fédéral de faciliter pécuniairement l'accès aux soins, malgré l'opposition souvent violente du Québec. L'exemple de Beveridge en Angleterre, la détermination de certains individus, la pression des syndicats, la situation financière de certains hôpitaux, les plates-formes électorales des partis politiques fédéraux, la capacité fiscale du gouvernement fédéral et plusieurs autres facteurs peuvent expliquer cette volonté. Toutefois, sans les actions du gouvernement CCF 9 en Saskatchewan et des gouvernements de quelques autres provinces de l'Ouest, le gouvernement fédéral ne se serait probablement pas engagé de façon aussi déterminée dans des batailles qui lui étaient électoralement dommageables au Québec. En effet, à la suite des actions des gouvernements de l'Ouest, les politiques de sécurité sociale devenaient très différentes entre provinces. Ceci rendait difficile la mobilité géographique à travers le pays et remettait en question l'existence même de standards nationaux et l'égalité des citoyens canadiens. Bref, la légitimité du Canada comme nation était en cause. Alors que le débat autour des assurance sociales avait été amorcé dès les années 1920, ce n'est qu'à la fin des années '50 qu'il devint politiquement impératif pour le gouvernement fédéral d'intervenir directement dans le financement des soins, en dépit de sa position constitutionnelle contestée en la matière 10. C'est ainsi qu'à la suite de l'introduction de programmes d'assurancehospitalisation dans les provinces de l'Ouest, le gouvernement fédéral se décida aussi à financer l'hospitalisation (1957); après que certaines de ces provinces eurent établi des plans publics d'assurance-maladie,

9 Le parti CCF (Cooperative Commonwealth Federation), maintenant appelé Nouveau Parti Démocratique, prit le pouvoir en Saskatchewan en 1944. Une de ses premières mesures d'importance fut l'introduction d'un plan d'assurance-hospitalisation.

10 Pour un examen des procédures légales autour de cette question, voir le volume I du rapport de la Commission d'enquête sur la santé et les services sociaux (L'assurance-maladie), Gouvernement du Québec, 1967, chapitre VI. 
le Parlement d'Ottawa en 1966 vota un programme public, universel et obligatoire d'assurance-maladie.

Au Québec, la province à l'époque la plus réactionnaire quant à ce genre de réformes, les programmes fédéraux furent reçus avec acrimonie par les partis politiques au pouvoir. Le gouvernement Duplessis refusa d'adopter le programme d'assurance-hospitalisation et l'administration Johnson fit de même concernant le plan d'assurancemaladie. En fait, jusqu'en 1970, ce sont les crises politiques que créèrent au Québec les législations successives du gouvernement fédéral qui seules déterminèrent les actions des gouvernements québécois en matière de santé. Quel que soit le parti politique au pouvoir, cette gestion des interventions fédérales prit toujours place dans un contexte de politique partisane. Pour chaque parti politique, l'objectif fut uniquement de maximiser la rentabilité électorale qu'il pouvait tirer de la lutte pour ou contre les plans fédéraux. L'ambition des partis, jusqu'en 1970, ne fut jamais d'utiliser les réformes dans les mécanismes de financement de soins comme leviers pour rationaliser l'organisation et l'administration des services de santé.

Toutefois, l'intrusion fédérale unilatérale en matière de santé força graduellement le gouvernement provincial à devenir le lieu où les divers problèmes et tensions sociales dans le domaine de la santé devaient être résolus. Revoyons rapidement les diverses étapes des interventions de l'État québécois au cours des années '60.

Alors que le Parlement fédéral votait unanimement le programme d'assurance-hospitalisation, l'Union Nationale au pouvoir à Québec s'obstinait à s'opposer à cette mesure, sous prétexte qu'elle violait les priorités québécoises. Pressé de toutes Parts cependant, Duplessis nomme la Commission Favreau qui ne fit jamais rapport à cause des élections de juin 1960. Le parti libéral prend alors le pouvoir avec une plate-forme réformiste où l'assurance-hospitalisation figure comme grande priorité. Voulant se donner rapidement une image de grand réformateur, il implante l'assurance-hospitalisation le 1er janvier 1961 dans un tel état de hâte et de précipitation que, malgré des efforts surhumains, la bureaucratie créée pour administrer le plan ne réussira à fonctionner efficacement que vers 1967 ce qui, plus tard, permettra à 
la Commission Castonguay-Nepveu de parler d'inefficacité administrative au Ministère québécois de la Santé.

Le même phénomène se produisit avec la Loi des Hôpitaux 11. À partir du moment où l'État devenait le tiers-payant des services hospitaliers. il devenait impératif que des règles et des normes soient édictées afin de réorganiser les conseils d'administration, de standardiser les procédures administratives, de réglementer les relations entre les hôpitaux et le gouvernement et entre les divers groupes travaillant dans l'hôpital. Confronté à une situation où les hôpitaux étaient administrés par des communautés religieuses dans le secret de leur ordre religieux et où les hôpitaux se faisaient concurrence pour des fonds, des médecins et des améliorations technologiques, indépendamment des besoins objectifs, de la communauté, l'État se devait de trouver les moyens d'ajuster le système hospitalier au nouveau système de financement publie. Divers scandales dans la gestion des fonds de certains hôpitaux forcèrent le gouvernement à intervenir et à voter la Loi des Hôpitaux en 1962. Cependant, ce n'est qu'en 1969 - à la suite de conflits entre le Collège des Médecins et les associations d'hôpitaux que les règlements qui devaient accompagner la loi pour définir l'organisation interne de l'hôpital furent édictées. À nouveau, la lenteur avec laquelle cette loi fut appliquée permettra à la Commission Castonguay-Nepveu de contester la qualité de la gestion du Ministère québécois de la Santé. Les contrôles publics de la croissance et de la direction de la croissance de l'ensemble de l'appareil hospitalier demeurèrent presque inexistants au cours de la décennie '60-'70. De même, aucune réglementation ne fut introduite afin de contrôler et contenir la croissance anarchique de la spécialisation en médecine, de

11 Même si elle ne fut que très chaotiquement implantée (v.g. en 1967, près du tiers des hôpitaux n'avait pas encore effectué le «transfert du patrimoine » exigé par la loi), cette loi est extrêmement importante. Elle fut. sous bien des aspects, le précurseur du «bill 65 » voté en 1971. De plus, elle contribua à laïciser la structure hospitalière du Québec presque aussi profondément que si les communautés religieuses avaient été unilatéralement expropriées du champ hospitalier. Le déclin du recrutement dans les communautés. combiné avec les dispositions de la loi relativement au contrôle des hôpitaux, incita presque toutes les communautés religieuses à abandonner leur pratique hospitalière. 
la quincaillerie médicale et du professionnalisme chez les paramédicaux.

En 1965, le gouvernement fédéral avait annoncé sa décision d'établir un programme d'assurance-maladie à travers le Canada. Bien qu'opposé, en paroles tout au moins, aux interventions fédérales, le gouvernement libéral - sans doute plus pour des raisons électorales que dans le but de rationaliser le système de santé - nomma un Comité, présidé par Claude Castonguay et chargé d'analyser la validité et les modalités d'application du programme fédéral. L'élection du gouvernement Johnson en 1966 repoussa de quatre autres années l'adoption de cette mesure.

Afin de retarder de façon élégante l'adoption du programme, l'administration Johnson créa la Commission d'Enquête sur la Santé et le Bien-Être Social (Commission Castonguay-Nepveu). Elle lui donna le mandat d'examiner l'ensemble des secteurs de la santé, du bien-être social et de la sécurité du revenu, sans aucune restriction quant aux questions qui devaient être examinées. Avec une telle carte blanche et un tel mandat, le gouvernement pouvait être assuré d'avoir «noyé le poisson » pour une longue période de temps. Toutefois, le cumul de divers événements - en particulier la grève des hôpitaux de 1966, la publication en 1967 du premier volume de la Commission qui favorisait l'introduction du programme fédéral, la grève des radiologistes en 1967, la taxe fédérale dite du " progrès social » 12 - finirent par forcer le gouvernement Johnson à s'impliquer davantage dans le domaine de la santé et à accepter d'introduire le programme d'assurance-maladie. Mais l'élection d'avril 1970 empêcha l'administration Bertrand - qui avait été le successeur de Johnson en 1968 - de le faire ${ }^{13}$.

12 En 1968, afin de financer le programme d'assurance-maladie, le gouvernement fédéral imposa une taxe supplémentaire de $2 \%$ du revenu des particuliers (avec un maximum de \$120.) Afin d'éviter les débats constitutionnels, on appela cette taxe, "taxe du progrès social ». Elle s'appliquait à tous les Canadiens, que leur province participe ou non au programme fédéral.

13 Une autre législation fut introduite en 1967 en réponse au plan fédéral d'assistance médicale. Comme ce plan ne visait qu'à venir en aide à ceux qui en avaient le plus besoin, les assistés sociaux, il ne rencontra pas d'opposition de la part de l'administration Johnson. 
La victoire du parti libéral et la nomination de Castonguay comme Ministre de la Santé, de la Famille et du Bien-être Social marqua la fin de la période ou le seul impératif d'action était de faire face aux interventions fédérales et d'utiliser la gestion des crises politiques ainsi créées uniquement comme monnaie d'échange dans l'obtention d'appuis électoraux. Après l'introduction du programme d'assurancemaladie et les querelles auxquelles elle donna lieu en 1970, l'action gouvernementale se déplaça vers l'organisation et l'administration des soins. Maintenant que le financement des soins était question réglée, de nouvelles priorités s'imposaient, en particulier la limitation de la croissance des coûts. Pour ce faire, il devenait impératif d'essayer de « rationaliser » l'allocation des ressources. Ce sera la tâche de Castonguay et de ses successeurs à partir de 1970.

Les années 1960 auront donc vu l'État québécois, après bien des délais et des luttes, se substituer graduellement au marché privé comme mécanisme d'allocation des ressources dans le domaine de la santé. Bien que cette présence de l'État fut en quelque sorte involontaire, elle institutionnalisa néanmoins le rôle de l'État en tant que responsable et de la santé de la population et des soins aux malades, chasse privée, jalousement gardée pendant si longtemps. Ainsi, les communautés religieuses délaissèrent le contrôle des hôpitaux, les services hospitaliers furent quelque peu standardisés à travers la province et on rendit gratuit l'accès aux soins hospitaliers, puis médicaux.

La réorientation des interventions de l'État québécois entraîna également une restructuration complète de l'organisation formelle des divers groupes composant la division du travail médical. En dix ans, le vieil ordre social où l'obéissance, le bénévolat et le consensus étaient la norme, devint, pour ainsi dire, une arène politique où des agents économiques rationnels s'affrontent pour défendre ou promouvoir les intérêts privés de leurs commettants. Les groupes qui, dans le passé, avaient contrôlé les mécanismes de prise de décision et avaient bénéficié d'un prestige et d'un pouvoir considérable, ou bien disparurent de la scène (communautés religieuses et, en partie, les compagnies d'assurance) ou bien s'organisèrent formellement en prévision des interventions gouvernementales (hôpitaux, médecins spécialistes). Les groupes qui avaient quelque chose à gagner (en statut, prestige, revenu, pouvoir, etc.) des interventions de l'État se mobilisèrent également 
(omnipraticiens, travailleurs hospitaliers, infirmier(ère)s, administrateurs, etc.). C'est ainsi, par exemple, que les médecins francophones qui, contrairement à leurs collègues anglophones, n'avaient jamais senti le besoin de créer un groupe de pression pour défendre leurs intérêts ${ }^{14}$ se regroupèrent dans deux syndicats médicaux, la Fédération des Médecins Omnipraticiens du Québec (FMOQ) et la Fédération des Médecins Spécialistes du Québec (FMSQ), qui, en temps opportun, n'hésitèrent pas à utiliser les techniques, si longtemps décriées, des syndicats ouvriers pour arriver à leurs fins. C'est ainsi également que, de cinq associations en 1960, les hôpitaux québécois finirent par se regrouper en une seule association, faisant ainsi paradoxalement du Québec une des seules provinces sans association propre aux hôpitaux catholiques ! C'est ainsi qu'infirmier(ère)s, physiothérapeutes, ergothérapeutes et diététicien(ne)s, firent grève, souvent plus d'une fois, alors que cela ne s'était jamais produit dans le passé. C'est ainsi enfin que la Confédération des Syndicats Nationaux connut l'accroissement quantitatif le plus grand de son histoire : $40 \%$ de ses quelque 100,000 nouveaux membres venant du secteur de la santé, phénomène qui devait rapidement modifier en profondeur son équilibre interne.

En somme, en une décennie, la scène québécoise de la santé se sera profondément transformée. Ainsi, l'État québécois aura acquis la capacité d'intervenir massivement, voire autoritairement, pour altérer l'ordre social traditionnel, et les groupes professionnels et institutionnels visés auront développé la capacité de défendre ou de promouvoir leurs intérêts personnels. La réorientation graduelle du rôle de l'État à la suite des initiatives fédérales et l'émergence de groupes d'intérêt bien structurés, ainsi que la légitimité presque charismatique dont jouissait Claude Castonguay, créaient, en 1970, une conjoncture politique exceptionnelle. Comme cela arrive rarement dans l'histoire d'une société, il devenait politiquement possible d'introduire démocratiquement des changements majeurs de l'ordre social dans le domaine de la

14 Alors que les médecins anglophones étaient regroupés dans le «Canadian Medical Association », les médecins québécois francophones n'avaient qu'une association de type culturel, l'Association des Médecins de Langue Française du Canada. C'est le Collège des Médecins et Chirurgiens de la Province de Québec qui défendait les intérêts de la profession dans les rares cas où c'était nécessaire. 
santé. Non seulement Castonguay lui-même avait été nommé Ministre, non seulement la scène politique avait été mise en place pour des interventions gouvernementales majeures, mais les grands principes d'une réforme fondamentale avaient été annoncés et acceptés par de nombreuses couches de la population. La Commission GastonguayNepveu avait en effet suggéré une réforme axée sur une nouvelle conception de la médecine, orientée vers la santé plutôt que vers la maladie, et fonction d'une refonte radicale de l'organisation et de l'administration des institutions et des professions de soins. Comme toutes les autres commissions d'enquête au Canada, elle avait suggéré une rationalisation bureaucratique des services de santé mais, contrairement à celles-là, elle y avait ajouté des objectifs et des idéaux beaucoup plus avant-gardistes.

\section{LE RAPPORT DE LA COMMISSION D'ENQUÊTE SUR LA SANTÉ ET LE BIEN-ÊTRE SOCIAL (COMMISSION CASTONGUAY-NEPVEU)}

$\underline{\text { Retour à la table des matières }}$

Malgré l'image de compétence et d'impartialité qui s'en dégagent, les commissions d'enquête, comités de recherche et task forces nommés par un gouvernement, sont d'abord et avant tout des organisations politiques. Elles ont le mandat de formuler des recommandations afin de renégocier l'allocation des ressources entre les divers éléments qui composent un certain secteur d'activité. Ces organismes sont créés parce que certains problèmes sont perçus comme suffisamment cruciaux et politiquement dangereux pour que les politiciens veuillent retarder les décisions et/ou parce qu'ils veulent obtenir des idées précises et susciter un débat autour des solutions que l'État devrait éventuellement apporter.

Dans tous les pays, la création d'un programme d'assurancemaladie universel, obligatoire et public, a inévitablement politisé le secteur de la santé et a forcé l'État, quels que soient les désirs des poli- 
ticiens, à faire quelque chose par rapport à la croissance phénoménale des coûts des services de santé et par rapport aux nombreux problèmes qui y sont coreliés. Lorsque l'État a décidé de financer le marché des soins traditionnellement privé, il est ainsi contraint d'essayer d'apporter des solutions aux tensions sociales et aux nombreuses contradictions inhérentes à la pratique contemporaine de la médecine. C'est pour cette raison qu'un très grand nombre de commissions d'enquête furent nommées au Canada à la fin des années '60 et au début des années '70.

Les membres de telles commissions ont une tâche bien précise. Ils doivent, premièrement, admettre, voire amplifier, l'existence des problèmes qui a créé leur rôle de formulateur de politiques et, deuxièmement, ils doivent postuler et convaincre le public de l'existence de solutions réalistes et efficaces à ces problèmes. Pour être légitimés, ces individus doivent donc faire appel à des symboles et à des sources de soutien qui peuvent cacher les intérêts qui sont objectivement servis. Ceci ne veut pas dire qu'ils sont menteurs, corrompus, malicieux ou manipulateurs, mais plutôt qu'ils doivent construire des représentations symboliques de leur rôle afin de mobiliser les appuis nécessaires à la légitimation de ce rôle. En d'autres mots, ils doivent développer une idéologie.

Pour cette raison, presque toutes les commissions d'enquête dans le domaine de la santé, à travers le monde occidental, doivent secréter la même idéologie technocratique : celle de vouloir rationaliser, par des méthodes bureaucratiques, les services de santé. Toutes prennent pour acquis que les services de santé sont désorganisés et inutilement fragmentés, qu'ils constituent un "non-système » qui doit - et peut - être remodelé en un «système » planifié, coordonné et intégré. On y affirme qu'avec des « objectifs » ultimes et intermédiaires bien délimités pour le «système », avec des distinctions claires entre les « niveaux de soins » et les " niveaux d'administration », avec une plus grande « expertise » dans la planification de normes « objectives » pour la " coordination » de l'allocation des ressources, avec une administration plus « rationnelle », avec des mécanismes formels qui garantissent "l'intégration » et la " responsabilité » des institutions de soins face au public, les services de santé seront significativement améliorés au bénéfice de tous et à un moindre coût. 
Ce faisant, on s'oppose à toute forme d'intervention de l'État qui perpétuerait la diversité et la compétition entre les professions et institutions de la santé, et qui supporterait aveuglément les grandes tendances de l'évolution contemporaine de l'économie médicale. On cherche, au contraire, à accroître le pouvoir de diverses bureaucraties sur les services de santé et à clarifier les lignes d'autorité et les règles du jeu. Au cours des années 1950 et 1960, l'anatomie et la physiologie du secteur de la santé ont été profondément modifiés. Celui-ci est devenu une industrie caractérisée par un ensemble hautement structuré d'entreprises industrielles, d'organisations de service, de professions, de rôles et de fonctions de plus en plus interdépendants. À cause de cette interdépendance, il y a une tendance à vouloir subordonner les tâches médicales de toutes sortes au contrôle d'organisations gigantesques, en particulier la bureaucratie gouvernementale et les hôpitaux. Ces organisations sont censées rationaliser l'allocation des ressources et des tâches en vue de maximiser l'efficacité économique globale du secteur de la santé. Toutes les commissions d'enquête, presque sans exception, ont cherché à accentuer cette rationalisation bureaucratique, s'opposant ainsi forcément aux praticiens à la base du système.

Une telle idéologie donne un rôle légitime à ceux qui ont reçu le mandat d'identifier les problèmes et de proposer des solutions : ils ont ainsi en main un diagnostic et une thérapie qui sont socialement valorisés. Mais le résultat tangible d'une telle idéologie peut être exactement l'inverse de ce qui est souhaité. Les nouvelles organisations, les nouveaux programmes et les divers chambardements administratifs suggérés, peuvent, en dernière analyse, pour des raisons que nous examinerons plus loin, renforcer les pouvoirs et les privilèges de groupes perçus comme la source de la désorganisation du système. Et ils peuvent contribuer à élaborer et compliquer davantage des structures bureaucratiques qui finiront par exacerber les problèmes de fragmentation, de désorganisation et de duplication que l'on prétendait résoudre.

Les recommandations de la Commission Castonguay-Nepveu au Québec vont exactement dans le même sens que les rapports élaborés ailleurs. On y redéfinit plusieurs des règles du jeu et on propose de rationaliser le système par un plus grand engagement des autorités 
publiques dans l'administration et l'organisation des services de santé, afin de véritablement coordonner, intégrer et planifier le personnel, les programmes et les établissements.

Ce rapport diffère toutefois à bien des égards des rapports équivalents dans les autres provinces. Les critiques formulées à l'égard de la situation passée y sont plus véhémentes : en particulier, les médecins et les hauts fonctionnaires du Ministère de la Santé y sont vertement critiqués. La rationalisation bureaucratique exigée y est beaucoup plus grande. Enfin, l'accent y est mis sur des idéaux et des buts sociodémocratiques d'une envergure sans parallèle au Canada. En lisant ce rapport, on a l'impression que le système de santé québécois est parmi les pires du Canada et que des réorganisations globales et massives sous l'égide de l'État sont absolument nécessaires pour le ramener à un niveau quelque peu acceptable. On a simultanément l'impression que, si les recommandations étaient suivies, le Québec aurait introduit une véritable révolution dans son secteur de la santé.

Les volumes IV et VII (tome 1) de la Commission 15 établissent en effet un dossier extrêmement convaincant sur la nécessité d'utiliser les changements dans les mécanismes de financement des soins comme leviers pour modifier en profondeur les schèmes de comportement dans le domaine de la santé. En plus de la rationalisation bureaucratique, quatre grands principes guident cette vision. On peut schématiquement les résumer ainsi :

1. Une médecine globale. Selon le rapport, l'objectif fondamental de la réforme de la santé doit être le développement d'une nouvelle conception de la santé, de la maladie et de la médecine. On y parle d'un modèle social ouvert par opposition au modèle médical fermé traditionnel. Parce que la mauvaise santé est souvent associée à toute une gamme de facteurs sociaux, tels la pauvreté et l'état général du milieu, on suggère de rationaliser le système autour d'un nouveau concept, celui de médecine globale. Cette médecine devra être fournie par une équipe multidisciplinaire de professionnels relativement

15 Rapport de la Commission d'enquête sur la santé et les services sociaux. volume IV (La Santé) Gouvernement du Québec, 1970 ; volume VII, tome I (Les Professions), Gouvernement du Québec. 1970. 
égaux, et responsables de la santé d'une population prédéfinie. Contrairement à la pratique médicale traditionnelle, celle-ci sera centrée sur la personne dans sa totalité plutôt qu'uniquement sur sa maladie spécifique. Et elle cherchera à identifier et à agir sur les conditions de vie et de travail qui conduisent à la maladie. En conséquence, la priorité devrait être donnée aux soins de première ligne (médecine générale et travailleurs sociaux) regroupant services préventifs, curatifs et de réhabilitation tant au niveau mental que physique. Les professionnels de la première ligne dirigeront - mais seulement en cas de nécessité absolue - les patients vers une série de services professionnels et hospitaliers de plus en plus spécialisés et complexes.

2. Un second objectif est de donner à la communauté et, dans une moindre mesure, aux établissements, l'autonomie nécessaire pour qu'ils puissent adapter à leurs besoins propres les priorités collectives dans la lutte contre la maladie. On suggère ainsi une décentralisation du pouvoir de décision vers les régions (en créant de mini-Ministères de la Santé) et vers certaines organisations (v.g. la formule du « budget global » devait rendre les hôpitaux responsables de l'allocation interne des ressources financières) à l'intérieur des priorités gouvernementales établies par un Ministère provincial complètement réorganisé.

3. Un troisième principe est d'ouvrir aux travailleurs de la santé et au publie tous les appareils formels de décision dans le domaine de la santé, de façon à ce que les décisions soient prises dans un contexte social d'ensemble plutôt qu'uniquement dans un contexte professionnel, financier, ou autre, comme c'était le cas dans le passé. On suggéra donc la participation, sous de multiples formes, des usagers et des travailleurs au contrôle des organisations qui les concernent.

4. Enfin, on affirme la nécessité d'une certaine égalisation des droits et des privilèges de ces travailleurs de la santé qui ont une formation technique ou universitaire et une certaine responsabilité par rapport aux patients, i.e. les « professionnels ». D'une part, on propose de distinguer, pour tout groupement professionnel, l'organisme responsable de la défense des intérêts économiques et sociaux des professionnels de l'organisme chargé de surveiller la pratique professionnelle. Les pouvoirs, obligations et privilèges de ces organismes doi- 
vent être standardisés, sous la surveillance de l'État. D'autre part, on suggère d'étendre le statut de " professionnel » à un ensemble d'autres groupes occupationnels, jadis totalement dominés par la profession médicale.

Ce sont ces principes qui créèrent, chez plusieurs, l'espoir d'une réforme fondamentale du secteur de la santé au Québec. Quand on le compare aux rapports ontariens, par exemple, le rapport de la Commission Castonguay-Nepveu apparaît effectivement extrêmement progressiste. On y propose non seulement une rationalisation bureaucratique des services de santé, mais aussi une ré-allocation des ressources en fonction de la santé plutôt que de la maladie ; un certain renversement du pouvoir jusque-là incontesté des médecins et une revalorisation des autres professionnels de la santé ; et, enfin, une prise en charge, réelle et effective, par la communauté, de ses besoins et de ses institutions et professions médicales. Les symboles ne sont toutefois pas synonymes de réalités. Comme nous le verrons, ce qui a été écrit à propos de la politique dans le domaine de la santé à New York s'applique au Québec également :

La politique sert à procurer à la fois des bénéfices tangibles à diverses élites et des bénéfices symboliques à la masse du public, émoussant ainsi les sources de contestation, détournant de leur objet les revendications potentielles et camouflant le véritable partage des bénéfices 16 .

16 Robert R, Alford, Health Care Politics : Ideological and Interest Group Barriers to Reform, Chicago et Londres, The University of Chicago Press, 1975, p. x. Pour un examen de la même hypothèse par rapport aux interventions gouvernementales dans d'autres secteurs, voir Murray Edelman, The Symbolic Uses of Politics (Urbana, University of Illinois Press, 1964) et Politics as Symbolic Action : Mass Arousal and Quiescence (Chicago, Markham Publishing Co., 1971). 


\section{LA RÉFORME}

$\underline{\text { Retour à la table des matières }}$

Castonguay devenant Ministre, en 1970, le rapport de la Commission d'enquête sur la santé et le bien-être social devint la perspective gouvernementale officielle sur la réforme de la santé. Dans aucune autre province canadienne, le point de vue formulé par une commission d'enquête ne devint si rapidement et si totalement la matrice intellectuelle fondamentale pour engendrer le changement.

Toute la législation dans les domaines de la santé et du bien-être social fut révisée, tout au moins en théorie, en fonction des principes socio-démocratiques mis de l'avant par la Commission. Une véritable « corrida législative 17 » prit place : Castonguay présenta à l'Assemblée Nationale réformes sur réformes, exposées dans leurs moindres détails et déduites de ce qui semblait être le cadre logique et philosophique extrêmement serré de la Commission.

Parmi les réformes qui retinrent le plus l'attention dans le domaine de la santé, on peut mentionner les suivantes.

- Le programme d'assurance -maladie dont la couverture fut graduellement élargie. - Suppression unilatérale de toutes les dépenses déjà approuvées et relatives aux immobilisations hospitalières. Cette mesure devait permettre de plus grands investissements dans les soins chroniques et de première ligne.

- Création du Ministère des Affaires Sociales (MAS) regroupant les anciens Ministères de la Santé, de la Famille et du Bien-être social et redéfinition de sa vocation. Au lieu d'être un distributeur de services comme c'était le cas du Ministère de la Santé précédent, le MAS se voit confier un rôle de leader, établissant les priorités, planifiant, met-

17 Gilbert Blain, « Le réforme doit prendre son second souffle », L'Union Médicale du Canada, tome 104, janvier 1975, p. 45. 
tant en route les programmes, les contrôlant et les évaluant. Sa structure interne est réorganisée en conséquence, d'une structure par programmes à une structure dite fonctionnelle.

- Création de nouvelles unités de soins de première ligne, les Centres Locaux de Services Communautaires (CLSC) où médecins, travailleurs sociaux et organisateurs communautaires, travaillant en collaboration, devaient essayer de développer une nouvelle médecine et de prendre en charge une population pour l'ensemble de ses besoins sociaux et de santé.

- Formation de Départements de Santé Communautaire (DSC) dans 32 hôpitaux du Québec, en remplacement des unités sanitaires et des services de santé des villes, afin de revaloriser le domaine traditionnellement dévolu à l'État, la santé publique (aujourd'hui appelé « santé communautaire »), et afin de commencer à inscrire dans la structure hospitalière, château-fort de la médecine curative spécialisée, une nouvelle vocation communautaire et préventive.

- Création d'organismes administratifs régionaux, les Conseils Régionaux des Services Sociaux et de Santé (CRSSS). Ces organismes n'ont au départ que des responsabilités consultatives.

- Adoption de la formule du budget global pour les hôpitaux, formule par laquelle le conseil d'administration de l'hôpital devait être seul responsable de l'allocation interne des fonds.

- Formation d'un organisme de contrôle des professions, ]'Office des Professions du Québec (OPQ). Les diverses organisations professionnelles voient leurs rôles et fonctions redéfinis et standardisés. Les syndicats professionnels sont clairement distingués des corporations professionnelles. Plusieurs groupes d'occupations reçoivent le titre de « profession » avec droit de pratique indépendante.

- Enfin, sur tous les conseils d'administration des établissements de soins, on prévoit la participation d'un certain nombre de représentants élus des travailleurs de l'établissement et des usagers. Des représentants du public sont également nommés aux conseils d'administration des corporations professionnelles. Enfin, afin de favoriser l'intégra- 
tion, chaque catégorie d'établissements (v.g. CLSC) est représentée dans les conseils d'administration des autres catégories d'établissements (v.g. hôpital) avec lesquels il a affaire.

En somme, un chambardement d'envergure prit place. On supprima certains organismes (unités sanitaires). D'autres furent créés (CLSC, DSC, CRSSS, OPQ). D'autres virent leurs rôles et fonctions redéfinis (MAS, organisations professionnelles). Plusieurs procédures de gestion furent modifiées et des responsabilités de gestion furent attribuées à des personnes autrefois totalement étrangères à ce travail. Et, finalement, la structure d'ensemble fut clarifiée sur papier, en distinguant ce qui étaient tâches d'exécution (le " réseau ») et tâches de conception et en reclassant l'ensemble des organisations du réseau en quelques catégories bien définies.

\section{LES RÉSULTATS DE LA RÉFORME}

\section{$\underline{\text { Retour à la table des matières }}$}

Et pourtant, malgré l'activisme législatif, malgré tous les remaniements organisationnels, malgré les efforts gigantesques apparemment déployés, le résultat tangible de ces interventions de l'État est presque l'inverse des espoirs et des attentes que la Commission CastonguayNepveu avait suscités. Un écart gigantesque s'est introduit entre ce qui fut promis et ce qui fut fait.

Il est difficile, voire présomptueux, quelques années seulement après les principales législations, d'essayer de faire sérieusement un bilan complet de la réforme ${ }^{18}$. On peut toutefois, à la lumière de l'expérience internationale, extrapoler à partir de la logique sous-jacente aux changements effectivement introduits ou non, les tendances de l'évolution future du secteur de la santé au Québec. La réforme semble

18 Pour des raisons d'espace, mon argument est ici réduit à sa plus simple expression. Le lecteur peut se référer au chapitre 5 de ma thèse pour plus de détails et les références appropriées. 
avoir renforcé plutôt que contrecarré ce qui constitue les principales caractéristiques d'évolution des institutions et professions sanitaires dans le monde occidental : introduction de nouveaux acteurs dans le système, les technocrates ou, plus largement, les « rationalisateurs bureaucratiques »; accroissement de la centralisation du processus de prise de décision dans les mains d'un petit nombre de catégories sociales, les « rationalisateurs bureaucratiques » et les professionnels de la santé ; croissance de la demande pour des soins curatifs et, en conséquence, croissance de la dépendance de la population par rapport à un appareil médico-hospitalier de plus en plus envahissant et coûteux ; accélération de la course à l'autonomie professionnelle et augmentation de la dominance, dans la division du travail sanitaire, de la profession médicale.

On peut également comparer la réalité de la réforme avec ses idéaux. Il ne fait aucun doute, comme nous le verrons, que ces idéaux n'ont pas été atteints, à court terme tout au moins. Il y eut beaucoup de chambardements et de transformations, sans que ceux-ci soient toutefois significatifs d'une réforme en profondeur. À plus long terme, il est certes possible qu'une évolution différente prenne place. Mais le phénomène important qu'on doit saisir ici, c'est que, si jamais au Québec s'institutionnalise une véritable médecine globale ou si jamais se développe une prise en charge réelle par la communauté de ses institutions et professions sanitaires, ces changements ne viendront pas de nouvelles interventions d'élites technocratiques à la recherche de structures rationnelles et cohérentes. Plutôt, comme nous le verrons en conclusion, ils découleront du fait que la question de la santé a été, au Québec, politisée à un point inconnu en Amérique du Nord, semant ainsi les germes de nouveaux mouvements sociaux et d'une nouvelle conscience par rapport à la santé, à la maladie et à la médecine.

On peut enfin comparer le résultat des réformes québécoise et ontarienne, l'Ontario et le Québec étant des provinces hautement comparables quant à leur dimension, leur structure politique et l'évolution antérieure des secteurs de la santé dans les deux provinces. La conclusion qui s'impose ici, c'est qu'au-delà des styles radicalement différents d'intervention de l'État, les changements introduits au Québec et en Ontario sont en fait très similaires. 
Revoyons, de façon extrêmement sommaire, le sort fait à chacun des grands axes théoriques de la réforme.

a. L'assurance-maladie eut vraisemblablement un certain impact redistributif. Elle rendit les services médicaux plus accessibles aux strates socio-économiques les plus démunies. Comme le montre un autre article de cette revue ${ }^{19}$, celles-ci reçoivent plus de soins médicaux et des soins qui sont peut-être plus appropriés à ses problèmes que dans le passé. Mais, le fait que l'on continue à payer les médecins à l'acte (contrairement à la volonté de la Commission), la structure des incitatifs financiers qui favorisent une sur-consommation des actes médicaux les plus coûteux et parfois les plus dangereux ${ }^{20}$, le développement d'une médecine dite de type défensif (avec consommation incontrôlée des techniques sophistiquées et potentiellement pathogènes de diagnostic), l'hypertrophie des urgences et des cliniques externes dans les hôpitaux où les soins sont inévitablement moins personnalisés et plus routiniers, sont tous des facteurs qui, directement ou indirectement, peuvent à moyen terme contribuer à diminuer, voire à inverser, les bénéfices réels que le programme d'assurance-maladie apporta.

b. Le concept de médecine globale se résuma, opérationnellement, à l'offre d'un plus grand éventail de services fournis par des organisations que des débats internes et externes ont jusqu'à maintenant presque paralysés. C'est le cas des CLSC qui furent, à l'origine, implantés de façon tout à fait chaotique sur le territoire, davantage en fonction d'une logique de récupération des cliniques populaires existantes et en réponse à des pressions politiques dans certains comtés, qu'en fonction d'une stratégie réfléchie pour arriver à véritablement transformer les soins de première ligne. La multidisciplinarité y engendra des conflits sclérosants, paralysant ainsi l'objectif d'un renouveau de la pensée médicale, qui devait être orientée vers la causalité multiple de la maladie. L'opposition systématique de la profession médicale aux CLSC en bloqua le développement. Pour contrecarrer l'impact possible de quelques 70 CLSC, la Fédération des Médecins Omnipraticiens du

19 Voir A. D. MacDonald et al., « Études sur l'assurance-maladie du Québec ».

20 Voir Luciano Bozzini et André-Pierre Contandriopoulos, « La pratique médicale au Québec : mythes et réalités ». 
Québec (FMOQ) encouragea la création de polycliniques privées où les médecins - bénéficiant de revenus extraordinairement élevés - sont rois et maîtres. Plus de 400 polycliniques furent ainsi ouvertes. C'est à l'intérieur de celles-ci, plutôt que dans les CLSC, que les médecins expérimentés acceptent de travailler. C'est aussi, peut-être dans une moindre mesure, le cas des DSC qui cherchent désespérément à se tailler une place à l'intérieur de l'appareil hospitalier en dépit de l'opposition ou de l'indifférence environnante. Il est loin d'avoir été encore démontré que les responsabilités curatives qu'on leur a attribuées (les urgences) ne contredisaient pas la volonté de développer une pratique de santé communautaire véritablement renouvelée. Enfin, les secteurs d'interventions de l'État qui sont partie intrinsèque d'une nouvelle conception de la médecine - la médecine du travail et les contrôles sanitaires de l'environnement - furent, en 1970, éliminés du champ des responsabilités du MAS et transférés au Ministère des Affaires Municipales. Bref, nous sommes encore très loin du concept de médecine globale prôné par la Commission Castonguay-Nepveu. Les récentes déclarations de l'ex-ministre Forget 21 à propos de la vocation des CLSC indiquent d'ailleurs que ce concept a peut-être été évacué des politiques gouvernementales.

c. Alors que, pour la Commission, la décentralisation administrative signifiait l'attribution d'un certain pouvoir réel à la communauté afin qu'elle décide elle-même de l'allocation des ressources en fonction des grandes priorités gouvernementales, pour le gouvernement, le concept de décentralisation signifia le regroupement d'unités antérieurement séparées, dans des corps régionaux consultatifs, afin de faciliter le commandement par le haut. En fait, la centralisation ne fut jamais aussi grande qu'après la réforme. Même l'introduction du « budget global » fut une décentralisation fictive puisque les activités financières des hôpitaux restèrent sous la surveillance détaillée du MAS dans 35 centres d'activités internes. On a dit aux hôpitaux qu'ils pouvaient gérer eux-mêmes leurs fonds mais la bureaucratie gouvernementale continue à surveiller les budgets hospitaliers dans leurs moindres détails.

21 Claude Forget, «Perspectives à l'égard des Centres Locaux de Services Communautaires », Ministère des Affaires Sociales, juillet 1976. 
d. C'est autour de l'opérationnalisation du principe de la participation des consommateurs et des travailleurs à la gestion des organisations qui les concernent, qu'il y eut le plus d'efforts originaux mis en branle. S'il est moins retentissant, l'échec de cette opérationnalisation n'en est pas moins réel. Les taux de participation aux élections, l'origine socio-économique élevée de plusieurs élus, les difficultés qu'éprouvent les élus d'origine plus modeste à s'exprimer dans un contexte hautement professionnalisé et bureaucratisé, en sont des indications claires. La principale difficulté tient à un vice fondamental de la conception même que s'est fait le gouvernement de la participation. Celle-ci fut en effet institutionnalisée davantage comme un mécanisme pourvoyeur de feedback aux autorités publiques, cooptant ainsi les leaders plus verbaux et les groupes plus critiques, que comme une délégation réelle de pouvoirs à un nouvel acteur dans le système. On donnait ainsi l'impression d'une ouverture du processus de décision mais, en fait, on facilitait et camouflait la domination des groupes les plus privilégiés de la société.

e. L'effort déployé pour réorganiser le monde des professions fut aussi impressionnant. Plusieurs contrôles formels ont été introduits afin de mieux protéger le publie. Mais l'objectif d'une certaine égalisation du statut des professionnels de la santé ne fut pas atteint. Certes, au plan légal, plusieurs occupations portent maintenant le titre de profession tout comme la médecine ; ils ont des droits comparables et ils sont régis par le même genre de mécanismes. Mais au plan social, la course à l'autonomie et aux prérogatives professionnelles qu'a stimulée cette législation s'est vu heurtée à la logique même d'un système de production où les médecins demeurent les patrons, des patrons souvent absents et quelque peu agacés par les contrôles bureaucratiques, mais des patrons néanmoins, qui ont autorité sur les services professionnels auxiliaires, qu'ils ne paient d'ailleurs pas. Ceci se manifeste sur plusieurs plans qu'il serait trop long d'examiner en détail ici. Donnons cependant quelques exemples : dans la structure hospitalière d'abord, où la profession médicale a gardé le contrôle des postes de gérance que lui enlevait la Commission; dans ses relations avec le gouvernement où, du simple fait qu'elle soit organisée et puissante, la profession médicale peut arriver à imposer facilement sa volonté, contrairement aux autres professions ; dans le système dans son ensemble, enfin. Bien que la profession médicale soit devenue un peu 
plus vulnérable aux contrôles extérieurs, elle a gagné un niveau de revenu et le contrôle de certains comités clés qui renforcent sa position de dominance et qui la distancent d'autant des autres catégories de professionnels.

f. Si les objectifs les plus fondamentaux de la réforme n'ont été que très partiellement atteints, ou pas du tout, qu'en est-il de la volonté de rationalisation bureaucratique qui constituait l'idéologie de base de la Commission ? Il ne fait pas de doute qu'il y eut, à ce chapitre, une certaine amélioration : un certain nombre des règles du jeu ont été clarifiées de même que les lignes d'autorité, mais pas dans les proportions escomptées. Par exemple, le MAS affirme qu'il planifie et coordonne, mais il y a plusieurs indications selon lesquelles, en dépit de l'expertise supposée plus grande de son personnel, malgré le vocabulaire technocratique qui est maintenant à la mode, malgré les structures internes du Ministère qui sont très rationnelles sur papier, il y aurait autant d'arbitraire dans l'établissement des nonnes, autant d'improvisation dans l'élaboration des politiques et autant d'incohérence dans leur implantation que dans le cas des Ministères de la Santé précédents, si fortement critiqués par la Commission. Ce manque de rationalité ne tient pas à une mauvaise gestion du MAS, mais plutôt à des obstacles structurels inhérents au genre de réformes introduites. En fait, il y eut tellement de chambardements administratifs et organisationnels qui furent opérés, qu'on peut se demander si les réformes n'ont pas augmenté la duplication, la fragmentation et la désorganisation qu'elles devaient solutionner, condamnant ainsi les gestionnaires de l'État à un rôle d'intendance. Prenons quelques exemples. Il y eut une réévaluation de la médecine de première ligne, en particulier par le moyen du tarif unique ${ }^{22}$, mais ceci entraîna le renforcement de la FMOQ, défendant avec âpreté le monopole de la profession contre tout changement du statu quo. Des CLSC furent créés mais, au lieu de devenir la seule porte d'entrée dans le système, comme c'était prévu, il y en a maintenant au moins cinq: les CLSC, les polycliniques, les bureaux des médecins en pratique solo, les cliniques externes des hôpitaux et les unités de soins familiaux des universités. Ceci multiplie les coûts, crée la confusion chez les consommateurs et rend la coordination très

22 Le tarif unique signifie que les médecins, omnipraticiens ou spécialistes, sont payés le même montant pour le même acte médical. 
difficile. Il y eut des efforts pour rationaliser les soins de deuxième ligne, en éliminant les dédoublements de services hospitaliers dans les régions et en essayant d'assurer un processus interne de prise de décision qui respecte davantage la complexité de la division du travail dans les hôpitaux. Mais, en fait, l'existence de deux lignes conflictuelles d'autorité à l'intérieur de l'hôpital est loin d'être une chose du passé : dans certains cas, les conflits ont été accentués. Et, malgré tous les contrôles, les hôpitaux sont des organisations trop complexes et trop gigantesques pour se soumettre facilement à la volonté d'une bureaucratie extérieure, même si c'est la bureaucratie gouvernementale.

En comparaison, les changements introduits en Ontario ressemblent curieusement à ceux mis en place au Québec. On les introduisit pourtant fort différemment. Les objectifs y furent beaucoup moins « révolutionnaires »: le seul objectif clairement explicité fut celui d'accroître la rationalité bureaucratique. Jamais, on n'y parla de médecine globale ou de participation ; et on ne laissa jamais sous-entendre qu'une certaine égalisation du statut des professionnels découlerait des changements organisationnels. De plus, les méthodes furent beaucoup plus souples : on utilisa des expériences pilotes ou des incitatifs financiers plutôt que la coercition législative. Par exemple, un certain nombre d'organismes régionaux ( "District Health Councils ») furent créés en Ontario et ils ont un pouvoir décisionnel, contrairement aux CRSSS québécois. On introduisit également dans les hôpitaux la formule du budget global, mais elle fut abandonnée parce que impraticable. Des organisations de soins communautaires, semblables aux CLSC, furent suscitées. Celles-ci ne comportent pas obligatoirement des travailleurs sociaux, ni la participation du public à leurs conseils d'administration, mais des médecins expérimentés y travaillent. Ces derniers acceptent volontairement d'être payés par la méthode de la capitation ou du salaire et de travailler dans une équipe médicale multidisciplinaire. Les unités sanitaires ontariennes sont également en train de se regrouper et de renouveler leur pratique de santé communautaire, dans la même direction que les DSC québécois. Mais elles ne sont pas rattachées aux hôpitaux. Le concept québécois de participation des citoyens n'a pas d'équivalent en Ontario, sauf pour les organismes de contrôle professionnel. Toutefois, le concept ontarien « d'équilibre des intérêts » sur les conseils d'administration n'est pas très différent de ce qui se passe en réalité au Québec, où très souvent 
seuls les groupes d'intérêt directement concernés participent aux élections. Ce qu'on doit saisir ici, c'est qu'avec des stratégies de réforme radicalement différentes, on arrive à des résultats qui sont fort semblables. Il est impossible d'affirmer a priori qu'une stratégie est meilleure qu'une autre : il existe des arguments pour et contre chacune des approches. Là n'est d'ailleurs pas la question. Le phénomène sociologique important, c'est que les différentes façons de faire au Québec et en Ontario aient conduit aux mêmes résultats d'ensemble, à court et moyen termes tout au moins.

En définitive, malgré les slogans qui, à un moment, on fait croire à une réforme fondamentale du secteur de la santé au Québec, cette réforme se résume, comme en Ontario, à une certaine réallocation des ressources entre divers groupes d'élites. Les technocrates gouvernementaux y ont considérablement accru leurs pouvoirs et leurs privilèges, leur idéologie, celle de la rationalisation bureaucratique, est au Québec devenue dominante. Les médecins se sont assurés la reproduction de leur domination professionnelle ainsi que, par l'intermédiaire de leurs syndicats, une amélioration très nette de leurs conditions de travail. Les autres professionnels de la santé ont vu leur statut légal s'améliorer quelque peu ; la consommation de leurs services s'est souvent accrue et de nouvelles possibilités d'emploi se sont ouvertes. Quant à la population dans son ensemble, elle y a acquis la gratuité des services, - ce qui est en soi une "victoire " extrêmement importante - mais pratiquement aucun autre bénéfice tangible. Les grands idéaux qui ont été mis de l'avant ne furent, pour la population, que les symboles rhétoriques d'un changement de la garde, des médecins aux technocrates gouvernementaux. 


\section{DE LA STRATÉGIE DES RÉFORMES}

$\underline{\text { Retour à la table des matières }}$

Alors que l'Ontario a introduit des réformes par des méthodes plus timides et avec des objectifs moins ambitieux en construisant à partir des arrangements institutionnels existants, le Québec, en moins de quatre ans, a changé l'ensemble de sa législation et une partie importante du cadre organisationnel des soins médicaux, sans atteindre un succès significativement plus grand que l'Ontario. Ceci soulève deux séries de questions précises. Nous essaierons d'y répondre séparément en proposant, très schématiquement, des modèles plausibles d'explication dans chaque cas.

La première question est de savoir pourquoi, au Québec, des idéaux socio-démocratiques furent mis de l'avant - médecine globale, décentralisation, participation et égalisation des statuts professionnels - et pourquoi des moyens plus coercitifs furent utilisés afin de les mettre en oeuvre. Comment se fait-il, en effet, que l'État québécois, après des années de passivité et de conservatisme, ait décidé d'agir de façon déterminée et en fonction d'objectifs clairement de gauche sur l'échelle des valeurs politiques nord-américaines ? En d'autres mots, pour reprendre la terminologie utilisée au début de ce texte, qu'est-ce qui explique le style de gestion ou de prévention des crises, la stratégie particulière de réformes de l'État québécois dans le domaine de la santé ? Trois réponses sont plausibles mais une seule résiste à l'analyse.

1. Une première explication possible est celle que retient le plus souvent le journalisme quotidien qui voit l'action politique comme la résultante du comportement d'individus, de leaders et de groupes d'intérêt. Ce serait à Castonguay et à son équipe qu'il faudrait attribuer le style particulier aux interventions de l'État québécois. Il y a une façon positive et une façon négative de voir les choses. Selon la première, Castonguay et son équipe auraient eu l'ambition personnelle de changer en profondeur le système de santé québécois. Après quatre ans 
d'un travail intense à l'intérieur d'une commission d'enquête, ils se seraient convaincus de la nécessité d'une réforme fondamentale. Toutes les alternatives ayant été considérées en commission, une fois au pouvoir, ils présentèrent lois après lois afin d'implanter cette réforme. Selon la version plus négative, Castonguay et son équipe auraient adroitement manipulé leur environnement afin de se négocier une position intéressante pour eux-mêmes et des personnes du même groupe d'élite. Ils se seraient faits du capital politique à l'intérieur de la Commission en noircissant quelque peu la scène médicale passée, en blâmant des groupes particuliers pour cette situation et en proposant des solutions de grande envergure. Capitalisant sur la légitimité ainsi obtenue, ils prennent le pouvoir et passent législation après législation pour maintenir leur image de grands réformateurs. Quelle que soit la variante choisie, une telle explication est très peu satisfaisante car elle repose sur des procès d'intention ouverts à toutes les interprétations ; d'autre part, elle néglige le fait social fondamental que le style particulier aux interventions étatiques dans le domaine de la santé fut hautement comparable au style de plusieurs autres réformes gouvernementales québécoises du début des années '60. En d'autres mots, une explication basée uniquement sur les individus impliqués dans la réforme, ne prend pas en considération le fait que ceux-ci sont, dans une grande mesure, les agents de forces sociales plus larges, qui influencent et déterminent leurs comportements et leurs attitudes par delà leurs volontés individuelles.

2. La culture québécoise est vue, par plusieurs, comme le facteur explicatif clé du style politique particulier au Québec. Caricaturalement, cette hypothèse pourrait se résumer ainsi : le fait que les élites canadiennes-françaises aient étudié, génération après génération, Descartes et Pascal, alors que les anglophones du même âge étudiaient Newton et Leibniz, ne peut que faire une différence énorme dans le style politique du Québec par comparaison avec les autres provinces. La culture québécoise étant plus "française », cartésienne et rationaliste, il est inévitable que toute réforme soit articulée sur des objectifs et des concepts globaux et qu'elle soit implantée par des méthodes rigides et universalistes plutôt que par des procédures souples et indirectes ("muddling through procedures»). Quoique séduisante, une telle ligne d'explication n'est pas suffisante. Il est vrai que les sociétés développent, en particulier par le contenu de leur système d'enseigne- 
ment, des visions du monde qui leur sont propres. Au Québec, le genre de mentalité développée dans les collèges classiques et dans les universités pourrait certes être une des causes de la reproduction, à travers l'histoire, d'une certaine façon de voir les choses, des élites religieuses traditionnelles aux élites laïques modernes. Mais ceci n'explique pas pourquoi cette façon de voir, cette forme, ait, ces dernières années, changé de contenu au point de devenir méconnaissable. De religieuse, apolitique, consensuelle et «tribale », pourrait-on dire, cette culture est devenue laïque, centrée sur l'État, social-démocrate, pluraliste et nationaliste. Pour comprendre ce changement, un autre modèle d'explication est nécessaire.

3. L'hypothèse la plus plausible et la plus complète réside dans les caractéristiques propres aux relations entre les classes sociales dans l'économie politique québécoise. C'est l'émergence d'une certaine petite-bourgeoisie nationale - qui trouve son intérêt dans l'expansion de l'État et dans l'accroissement de la légitimité de l'appareil d'État - qui est la caractéristique fondamentale de ces relations et qui explique le mieux la stratégie de réformes particulière au Québec. Nous ne pourrons examiner ici cette hypothèse qu'à très grands traits.

Partout au Canada, les années '50 et '60 ont connu une expansion considérable des secteurs publics provinciaux, à cause de la prise en charge par l'État des secteurs de la santé, de l'éducation et du bienêtre, et à cause du plus grand engagement de l'État dans l'économie (création et nationalisation d'entreprises, formation de bureaux de planification économique, etc.). Dans toutes les provinces, cette expansion fut associée à un changement dans les institutions dominantes, de religieuses et rurales à laïques et urbaines. Au Québec, cependant, contrairement aux autres provinces, parce que cette expansion eut lieu dans un contexte politico-économique différent, elle altéra profondément la matrice des relations entre les classes.

On peut caractériser cette économie politique par les éléments suivants. Premièrement, depuis la fin de la dernière guerre mondiale, des changements structuraux profonds se sont produits dans l'économie québécoise, les emplois de cols-blancs et de travailleurs manuels spécialisés deviennent de plus en plus nombreux par rapport aux emplois de manoeuvres et d'agriculteurs. En conséquence, la fraction franco- 
phone de la population québécoise a connu une mobilité ascendante forcée considérable. Ceci eut pour résultat qu'une proportion croissante de la jeune population entra au collège et à l'université. C'est vers le milieu des années '60 - et le phénomène s'accéléra par la suite qu'une masse de jeunes gens instruits commença à se chercher des emplois. Deuxièmement, le secteur privé de l'économie québécoise est moins dynamique que celui de l'Ontario ou de la Colombie Britannique, en termes de productivité, de sa capacité de susciter de nouveaux investissements et de créer de nouveaux emplois. Troisièmement, très peu de francophones ont jusqu'à maintenant réussi à pénétrer les échelons supérieurs et intermédiaires du secteur privé de l'économie, même à compétence égale avec les anglophones.

Comme les francophones québécois veulent travailler dans leur propre province, quelles que soient les possibilités d'emploi, contrairement aux autres Canadiens, cette conjoncture politico-économique donna à l'expansion de l'État québécois des années '60 et '70 une dynamique unique à travers le Canada. L'État devenait un des seuls pourvoyeurs d'emploi pour cette fraction croissante de la population francophone ayant une formation universitaire. Sans l'expansion de l'État, l'écart entre anglophones et francophones au Québec se serait considérablement accru, les échelons supérieurs et intermédiaires d'une économie privée, d'ailleurs assez faible, étant fermés aux francophones. Ainsi, seul l'État québécois pouvait constituer la base institutionnelle pour la création d'emplois prestigieux et rémunérateurs pour les francophones instruits.

En d'autres termes, ces derniers ont une vocation forcée vers le secteur étatique de l'économie, i.c. le gouvernement, ses entreprises et régies autonomes, et les industries ou organismes dépendant directement ou indirectement de l'État. Les Canadiens français ayant une formation universitaire constituent une classe sociale au sens où leur capital académique leur font partager une capacité identique sur le marché de l'emploi et leur fournit des intérêts communs à voir l'État créer des emplois intéressants pour eux. Bien que cette classe soit constituée des individus que les statisticiens du recensement nomment "professionnels et techniciens ", ce n'est pas simplement un agrégat statistique. C'est une collectivité rivée à l'ordre social de façon très systématique : par le même capital symbolique qu'apportent ses mem- 
bres sur le marché du travail. On peut l'appeler une « nouvelle classe moyenne » ou une « nouvelle petite-bourgeoisie ».

Afin de maintenir la croissance de l'économie et des niveaux de chômage relativement bas, l'État québécois n'a pas d'autre choix que d'appuyer l'accumulation du capital dans des mains étrangères, soit directement (v.g, subsides à des multinationales), soit indirectement (v.g. socialisation de certains coûts de production, travaux publics pour compenser le manque de dynamisme du secteur privé de l'économie). Cette réalité est contraire aux intérêts immédiats de la plupart des fractions de la nouvelle classe moyenne. Mais celle-ci ne peut s'y opposer - autrement qu'en paroles - sans mettre en danger l'État québécois lui-même. À l'occasion, en socialisant les coûts d'achat ou de création de certaines entreprises, et en nationalisant les profits, certaines fractions de cette classe peuvent réussir à acquérir privément le pouvoir d'allouer les ressources dans l'économie manufacturière. Mais en général, c'est dans les secteurs d'activité où l'État a une plus grande autonomie - les services en particulier - que cette nouvelle classe moyenne recherche avec le plus de force une dominance locale, limitée mais réelle.

Toute action qui étend, quantitativement et qualitativement, l'emprise de l'État sert les intérêts objectifs de cette classe. Celle-ci a ainsi développé une culture politique qui valorise les réorganisations globales et visibles, axées, dans le contexte hautement politisé du Québec, sur des idéaux socio-démocratiques et nationalistes plus larges. Même si des actions de l'État peuvent entraîner à court terme des conflits entre des fractions de cette nouvelle classe moyenne (v.g. entre " professionnels » et technocrates), c'est son intérêt commun de voir se maintenir ou s'accroître l'activisme gouvernemental et de suggérer ainsi à l'ensemble de la population québécoise que l'État québécois est son seul levier collectif.

Dans ce contexte, quand pour une raison ou pour une autre, un groupe d'élites technocratiques - tels les membres dominants de la Commission Parent ou de la Commission Castonguay-Nepveu - apparaît sur la scène et formule des politiques visant à produire des chambardements organisationnels très visibles et fonction de cette culture politique, il reçoit automatiquement beaucoup d'appuis et il pénètre 
facilement l'appareil d'État. Quels que soient les conflits que peuvent engendrer à court terme de tels groupes à l'intérieur de la nouvelle classe moyenne, c'est l'intérêt de cette classe dans son ensemble de fournir à ces élites technocratiques une légitimité et un pouvoir très grand. Car elles contribuent à l'expansion quantitative et qualitative de l'État. De tels groupes reçoivent ainsi, à l'intérieur du Cabinet et de l'Assemblée Nationale, carte blanche pour introduire rapidement des réformes globales.

Dans un contexte différent, tel l'Ontario, des groupes comparables n'ont jamais reçu les mêmes pouvoirs, ni la même légitimité. Une nouvelle classe moyenne existe aussi en Ontario. Mais l'État Ontarien ne constituant pas la seule base institutionnelle de sa survivance, il n'y a pas de forces sociales qui incitent à soutenir presque inconditionnellement les élites technocratiques dont l'objectif est d'étendre l'emprise de l'État. En d'autres mots, dans un contexte comme l'Ontario, il est impensable qu'un groupe d'élites comme la Commission CastonguayNepveu en arrive à contrôler complètement l'activité gouvernementale dans un certain secteur et à y imposer ses propres idéaux, sa propre rationalité et sa propre façon de faire.

\section{DE L'ÉCART ENTRE LES IDÉAUX ET LA RÉALITÉ DE LA RÉFORME}

$\underline{\text { Retour à la table des matières }}$

Ceci soulève une deuxième série de questions. Comment est-il possible que des élites technocratiques, bénéficiant d'une très grande légitimité sociale, et ayant créé des espoirs de réforme fondamentale alors qu'elles travaillent dans une commission d'enquête, deviennent incapables, deux ans plus tard, lorsqu'elles sont au pouvoir, d'implanter une telle réforme ? Comment se fait-il que, comme une loi inexorable de la nature, les interventions de l'État dans le domaine de la santé semblent déterminées à maintenir, voire à renforcer, les pouvoirs et les privilèges des institutions et des professions sanitaires existantes, 
même si la conjoncture politique est très favorable au changement comme c'était le cas au Québec à la fin des années '60 ? Bref, qu'est-ce qui explique l'écart gigantesque entre les buts et idéaux de la réforme et leur réalité opérationnelle ?

Robert Alford a récemment élaboré une théorie 23 qui fournit un premier élément de réponse à ces questions. Très succinctement, il suggère que la caractéristique fondamentale de la politique dans le domaine de la santé depuis la dernière guerre est l'émergence d'une nouvelle idéologie de la santé et de l'organisation des soins, celle de la rationalisation bureaucratique et, corollairement, l'apparition de ce qu'il appelle un nouveau " groupe structural d'intérêts », les rationalisateurs bureaucratiques. Ceux-ci cherchent à réorganiser le système des services selon une logique managériale et à étendre leur contrôle sur l'organisation des services de santé en plaçant les fournisseurs de services, i.e. les professionnels de la santé, sous le contrôle des conseils d'administration des hôpitaux, des conseils régionaux et des bureaucraties gouvernementales. Ce groupe est à la fois en conflit et en symbiose avec un autre groupe, les monopoleurs professionnels. Ceux-ci, pour diverses raisons, ont historiquement obtenu un monopole sur les services de santé et sur la définition des conditions sociales de leur travail. La « santé » est devenue leur propriété exclusive et ils ont réussi à définir les problèmes de santé comme des "maladies ", exigeant un diagnostic et un traitement immédiat. En opposition aux rationalisateurs bureaucratiques, ils veulent maintenir le cadre institutionnel qui garantira la continuation et la reproduction de leur autonomie professionnelle et autres privilèges. Ils veulent ainsi ériger des barrières afin de protéger leur contrôle de la recherche bio-médicale, de l'enseignement et des soins. Une des caractéristiques fondamentales des combats sociaux dans le domaine de la santé est, selon Alford, ce conflit permanent entre rationalisateurs bureaucratiques et monopoleurs professionnels. Mais, simultanément, il existe une symbiose presque totale entre ces groupes. Ni un ni l'autre n'a intérêt à s'affronter véritablement, en ce sens que l'élaboration de contrôles bureaucratiques en haut de la structure est justifiée par le besoin de coordonner l'expansion des unités de service en bas de la structure. Chaque groupe préfère ainsi additionner ses propres besoins organisationnels et tech-

23 Robert Alford, op. cit., 1975. 
nologiques, de sorte que le système est perpétuellement en crise, les rationalisateurs bureaucratiques dénonçant la montée des coûts et l'aberration des formes organisationnelles existantes, les monopoleurs professionnels dénonçant les dangers de l'intrusion de ces derniers pour la qualité des soins et la relation patient-médecin. Ils se livrent ainsi une lutte dont ils sont, en dernière analyse, les seuls bénéficiaires et non la population.

Cette théorie décrit fort bien le genre de batailles qui se sont déroulées au Québec et elle explique plusieurs des échecs de la réforme. Le conflit et la symbiose entre les deux fractions de la nouvelle classe moyenne que constituent les rationalisateurs bureaucratiques et les monopoleurs professionnels, expliquent en partie pourquoi les objectifs de rationalisation bureaucratique ne furent que très partiellement atteints, Dans une économie de marché, comme celle de la santé, propriété d'entrepreneurs professionnels et industriels, la rationalisation bureaucratique ou, en d'autres mots, la planification sont entreprises bien illusoires.

Mais la théorie d'Alford ne peut pas rendre compte du fait qu'en dépit de la meilleure conjoncture concevable, Castonguay et son équipe ne réussirent pas à implanter au moins partiellement certains des grands idéaux de la réforme. En 1970, il semblait qu'autre chose que des ajustements mineurs au système des soins et que de légères modifications dans l'allocation des ressources, prendrait place au Québec, Il semblait qu'une nouvelle approche de la santé et de la maladie, et du contrôle et de la planification des institutions et professions sanitaires, serait opérationnalisée. Comme nous l'avons vu plus haut, le gouvernement québécois avait cessé d'être à la remorque du gouvernement canadien dans le domaine de la santé, et il s'était imposé comme le principal mécanisme d'allocation des ressources. De plus, la Commission Castonguay-Nepveu avait développé un projet de réforme qui avait été acclamé par de nombreuses couches de la population et avait doté Castonguay, devenu Ministre, d'un grand prestige et d'une très grande légitimité. Un autre modèle d'explication est donc nécessaire.

Celui-ci renvoie au rôle même de l'État dans une société capitaliste. L'État est l'appareil qui détient la légitimité nécessaire à la solu- 
tion des problèmes sociaux ; c'est une structure qui, à la fois, reproduit le système des classes et fournit un lieu pour la lutte des classes. C'est le lieu où, ultimement, les tensions sociales doivent être résolues et les contradictions réduites, pour maintenir l'ordre et l'harmonie sociale. L'État gère ainsi avec précaution les crises et il développe des stratégies à long terme de prévention des crises, dans le but de remplir deux fonctions contradictoires : soutenir l'accumulation du capital et légitimer les conséquences sociales de cette accumulation. L'État doit simultanément maintenir ou améliorer les conditions pour une accumulation profitable du capital - afin de maintenir le niveau de vie, créer des emplois, etc. - favorisant ainsi ceux qui profitent de cette accumulation, et maintenir ou améliorer les conditions pour l'harmonie sociale ${ }^{24}$.

Un tel rôle impose - et j'ai exposé cette hypothèse plus en détails ailleurs 25 - une logique précise aux interventions de l'État dans le domaine de la santé, une logique qui contient et contraint toutes les actions de l'État quelle que soit la volonté des acteurs impliqués. L'État, dans une société capitaliste, ne peut pas contrecarrer la logique selon laquelle une bonne santé dépendrait presque uniquement de la production, de la distribution et de la consommation de marchandises. Quand l'État intervient, il ne peut pas agir contre l'équation entre guérison et consommation qui est inscrite au cœur même du système de production capitaliste et par laquelle les problèmes de santé sont transformés en problèmes particuliers de consommation sur un marché économique précis.

C'est le paradigme dominant dans la médecine moderne, le modèle « engineering », qui institutionnalise cette transformation. Celui-ci

24 Pour une élaboration de cette théorie de l'État, voir Clauss Offe, « The Theories of the Capitalist State and the Problem of Policy Formation », in Leon N. Lindberg et al. (eds.), Stress and Contradiction in Modern Capitalism, Lexington Books, 1975, pp. 125-144 ; voir aussi : James O'Connor, The Fiscal Crisis of the State, New York, St-Martin's Press, 1973 ; Alan Wolfe, "New Directions in the Marxist Theory of Politics », Politics and Society, 1974, 4 (2), pp. 131-159.

25 Marc Renaud, «On the Structural Constraints to State Intervention in Health », International Journal of Health Services, volume 5, no 4, 1975, pp. 559-572. 
décortique le corps humain en parties et en fonctions, telle une machine que l'on pourrait démonter et réassembler si ses structures et ses fonctions étaient bien comprises. Historiquement, cette façon de voir conduisit à la croyance que la découverte des agents étiologiques propres à une maladie et la compréhension de la réponse du corps à ces agents, permettrait d'intervenir thérapeutiquement de façon efficace, par des méthodes chirurgicales, chimiques ou électriques. Dans la population, ce paradigme a créé la croyance que chaque problème de santé pouvait être «traité »ou " prévenu » par une technologie, un personnel et un cadre organisationnel précis. On a ainsi fait croire à l'efficacité thérapeutique presque miraculeuse des services de santé, on a évacué épistémologiquement la composante sociale de l'étiologie des maladies, on a isolé l'individu face à sa maladie avec l'aide d'experts de plus en plus professionnalisés et on a favorisé la consommation d'équipements de plus en plus complexes et la prescription de médicaments de plus en plus différenciés, rendant ainsi la solution apportée aux maladies -lesquelles prennent en bonne partie leurs sources dans l'industrialisation capitaliste 26 - compatible avec l'organisation capitaliste de l'économie.

Quand l'État intervient, il ne peut que produire des changements qui renforcent, ou tout au moins maintiennent, l'allocation des ressources vers le marché des techniques thérapeutiques, du personnel et des organisations de soins, et vers des moyens de prévention technologiquement sophistiqués, sans être en mesure de produire les conditions sociales qui seraient favorables à une amélioration significative de l'état de santé de la population. Quelle que soit leur idéologie et quelle que soit la conjoncture, c'est là la limite au-delà de laquelle les rationalisateurs ; bureaucratiques ne peuvent aller, sans contredire le rôle même de l'État dans la société capitaliste.

C'est ainsi qu'il est relativement facile d'introduire un programme d'assurance-maladie puisque celui-ci ne fait que soutenir le marché

26 Voir par exemple, John Powles, « On the Limitations of Modern Medicine », Science, Medicine and Man, 1973, 1 (1) pp. 1-30 ; Joseph Eyer, « Hypertension as a Disease of Modern Society », International Journal of Health Services, 1975, 5 (4) pp. 539-558 ; Ingrid Waldron, « Why do Women Live Longer than Men ? », Journal of Human Stress, vol. 2, mars et juin 1976. 
économique de la santé, alors même que de nombreux consommateurs ne peuvent plus y participer. Les débats et les luttes qui entourent souvent la création de tels programmes ne sont que l'expression publique de la stratégie de négociation de divers groupes d'intérêts : c'est le rituel politique nécessaire pour que ces groupes améliorent ou maintiennent leurs pouvoirs et leurs privilèges. Une redistribution différente des ressources entre les diverses institutions composant ce marché représente également des changements cohérents avec la logique du système. Mais, c'est une toute autre histoire lorsqu'il s'agit d'introduire des réformes qui cherchent à sortir les problèmes de santé de l'orbite de la production, de la distribution et de la consommation de marchandises. Les concepts de "médecine globale » et de "participation » au Québec représentaient un effort, très limité certes mais réel, pour créer une nouvelle vision de la santé, de la maladie et de la médecine, pour réduire le monopole de la profession médicale sur la définition et le traitement des maladies, et pour rendre la communauté responsable des institutions et des professions sanitaires. Mais l'ordre social capitaliste dans le domaine de la santé a réduit ces concepts à n'être que des symboles rhétoriques pour légitimer une redistribution des ressources entre divers groupes d'élites.

\section{CONCLUSION}

$\underline{\text { Retour à la table des matières }}$

Le Québec a essayé d'aller beaucoup plus loin dans ses réformes de la santé que les autres provinces canadiennes. On y a développé de grands idéaux et on s'est appliqué à les implanter dans les législations. Ce faisant, l'État québécois a emprunté un style politique comparable à celui qui a présidé aux autres réformes de la Révolution Tranquille. Ce style peut largement être expliqué par l'émergence au Québec d'une nouvelle classe moyenne en quête d'emplois appropriés à sa formation scolaire. Au Québec, ceux qui ont un quelconque capital académique ont un intérêt objectif - enraciné profondément dans l'économie politique du Québec - à voir l'État québécois subordonner à son autorité de plus en plus de secteurs de l'économie et paraître ainsi le seul levier collectif. Dans ce mouvement par lequel l'État se défi- 
nit comme capable de réduire l'impact du colonialisme ou de l'impérialisme extérieur, de grands objectifs socio-démocratiques s'élaborent et l'appareil législatif de l'État se met en branle pour les réaliser. Mais ces objectifs se heurtent à la logique même d'une société où l'État n'a qu'un rôle de médiateur et de gestion des crises, et où la préservation et l'amélioration de la santé sont définis comme dépendantes du développement et de la consommation des techniques médicales. Cette logique fait en sorte que les discours planificateurs et rationalisateurs dans le domaine de la santé, de même que les grands idéaux mis de l'avant, ne sont guère plus qu'une arme aux mains d'élites technocratiques, utilisée pour des combats stériles contre la profession médicale et pour camoufler aux yeux du public le véritable partage des bénéfices. À long terme toutefois, les conséquences des interventions de l'État peuvent être différentes de celles observables à court et à moyen termes.

L'histoire est en effet processus dialectique. On ne paie pas impunément une population en monnaie de singe, sans produire des mouvements sociaux qui contestent la logique des interventions de l'État dans le domaine de la santé. Et, on ne développe pas impunément un appareil médico-hospitalier sans que ne se produise une crise fiscale 27 qui force l'État, dans ses interventions, à dépasser le seuil de la rationalisation bureaucratique.

Lorsqu'il devient clair que les coûts continuent à augmenter, quelles que soient les réformes, et que même la capacité fiscale de l'État est mise en péril, il est inévitable que les autorités publiques commencent à se poser des questions sur l'efficacité thérapeutique réelle du marché de la santé et sur ce que devraient être les priorités gouvernementales. En d'autres mots, le développement d'un appareil médicohospitalier constamment plus assoiffé d'expansion, ne peut, à long terme, que forcer l'État à se sortir d'un marché qu'il a lui-même contribué à étendre ${ }^{28}$. Le rapport du ministre fédéral Marc Lalonde, Nou-

27 Cette question est explorée dans le travail de James O'Connor (op. cit., 1973).

28 J'élabore davantage cette hypothèse dans « Crise de la médecine et politiques de santé : les leçons de l'histoire », Possibles, 1977, 1 (2), p. 33-49. 
velle perspective de la santé des Canadiens 29 est d'ailleurs extrêmement significatif à cet égard. On y soutient que les actions sur les facteurs individuels de risque (cigarette, alimentation, etc.) et - ceci est plus vague - sur l'environnement doivent maintenant devenir prioritaires, au détriment des services dits de santé. Pour le gouvernement fédéral - celui là même qui fut l'initiateur principal des plans d'assurance-hospitalisation et d'assurance-maladie - ce rapport représente un renversement total de perspectives.

Présentement, c'est le problème des coûts de la santé qui est au cœur des actions du gouvernement fédéral. C'est la même chose au Québec. Mais il y a plus. La contradiction entre ce qui fut promis et ce qui fut fait, entre les symboles et la réalité de la réforme, constitue la base d'une nouvelle série de crises à partir desquelles pourraient finir par émerger des réformes fondamentales.

Sur la base des frustrations accumulées chez ceux dont les espoirs ont été manipulés - travailleurs dans les CLSC, usagers élus sur les conseils d'administration, professionnels para-médicaux, etc. - peuvent prendre naissance des attaques de plus en plus véhémentes contre les pouvoirs et les privilèges de certains groupes dans le secteur de la santé. Ces attaques peuvent elles-mêmes forcer l'État, qui doit maintenir l'harmonie sociale, dans des directions différentes de celles qu'il a prises dans le passé.

Il y a encore plus. La visibilité et la rhétorique autour des actions de Castonguay, de même que tous les chambardements administratifs et les changements organisationnels qu'il a produits ont, au Québec, profondément politisé les enjeux relatifs à la santé et aux soins. Dans un sens général, la stratégie québécoise de réformes a, à long terme, arraché ces enjeux des seules mains des « experts ». En conséquence, les mouvements sociaux en matière de santé reçoivent maintenant une attention incomparable de la part de la presse, mettant ainsi rapidement en action les mécanismes de gestion et de prévention des crises dans l'État. Contrairement à la situation existante il y a quelques années à peine, plus un jour ne passe sans que la presse ne parle d'indus-

29 Marc Lalonde, Nouvelle Perspective de la santé des Canadiens, Gouvernement du Canada, 1974. 
tries polluantes, d'accidents sur les chantiers de construction, de grèves sur des questions de sécurité et de santé au travail, de la qualité des aliments, etc. En fait, un journalisme inquisiteur et acerbe semble apparaître sur la scène québécoise de la santé.

C'est l'ensemble de ces facteurs qui éventuellement pourront conduire à une véritable réforme du secteur de la santé. Grâce aux mouvements de la base soutenus par les média, grâce aux demandes de ceux qui furent directement frustrés par les actions du gouvernement québécois au début des années '70 et à cause des préoccupations sans cesse renouvelées des gestionnaires gouvernementaux pour les coûts de l'appareil médico-hospitalier, il est possible qu'enfin les vraies questions soient posées et que l'État soit forcé d'intervenir plus directement sur les facteurs pathogènes liés à la croissance industrielle capitaliste, améliorant ainsi véritablement la qualité de la vie et l'état de santé de la population.

\section{RÉSUMÉ}

Au début des années 1970, le gouvernement québécois intervint de façon massive afin de réformer le financement, l'organisation et l'administration des services de santé, en fonction de grands objectifs socio-démocratiques. Bien que le programme d'assurance-maladie eut un impact redistributif important, les autres aspects de la réforme n'apportèrent au public guère plus que des bénéfices symboliques, camouflant ainsi le véritable partage des bénéfices entre divers groupes d'élites. Replaçant cette situation dans le contexte des autres réformes de la Révolution tranquille et la comparant à la situation ontarienne, l'auteur souligne le caractère idiosyncratique des stratégies québécoises de réforme et leur incapacité d'atteindre les idéaux mis de l'avant. Il suggère que ces phénomènes renvoient aux caractéristiques propres aux rapports entre les classes sociales au Québec et à l'existence de contraintes structurelles encadrant les interventions de l'État dans une société capitaliste. 


\section{SUMMARY}

At the beginning of the 1970's, the Quebec government massively intervened in the reformation of the financing, organization and administration of health care services, as a function of broad social democratic objectives. Although the health care insurance programme had an important impact regarding the redistribution of health care, the other aspects of reform were little other than symbolic benefits, bluffing the true allocation of rewards amongst diverse elites. Placing this situation within the context of other reforms from the Quiet Revolution, and comparing them to the situation in Ontario, the authour underlines the idiosyncratic character of Quebec reform strategies, and their inability to live up to their stated goals. He suggests that such a phenomenon is attributable to the relationships between social classes in Quebec and to the existence of structural constraints to State interventions in health in a capitalist society.

\section{RESUMEN}

El gobierno de Québec al comienzo de los años 70 hizo una intervención importante en los servicios de salud, con el fin de reformar su finanzamiento, su organización y su administración en función de grandes objetivos socio-democráticos. Aunque el programa de seguro de enfermedad tubo un impacto distributivo importante, los otros aspectos no aportaron nada más que beneficios simbólicos, camuflando así el verdadero reparto de los beneficios entre los diversos grupos de élites. El autor coloca esta situación dentro del contexto de las otras reformas de la revolución tranquila, la compara con la situación de Ontario, muestra el carácter idiosincático de las estrategias quebequences de reforma y de su incapacidad a alcanzar los idéales que habian sido puestos por delante. Sugiere que esos fenómenos provienen de las características propias de las relaciones entre las clases sociales de Québec y de la existencia de frenos estructurales a las intervenciones del estado en una sociedad capitalista.

Fin du texte 